\title{
Expression of LRIG1 and LRIG3 correlates with human papillomavirus status and patient survival in cervical adenocarcinoma
}

\author{
SUSANNE MULLER ${ }^{1 *}$, DAVID LINDQUIST $^{2 *}$, LENA KANTER $^{3}$, CARMEN FLORES-STAINO $^{4}$, \\ ROGER HENRIKSSON ${ }^{2}$, HÅKAN HEDMAN ${ }^{2}$ and SONIA ANDERSSON ${ }^{1}$
}

\author{
${ }^{1}$ Department of Women's and Children's Health, Division of Obstretics and Gynocology, Karolinska Institute, \\ Elevhemmet H2:00, Karolinska University Hospital Solna, SE-171 76 Stockholm; ${ }^{2}$ Oncology Research Laboratory, \\ Department of Radiation Sciences, Umeå University, SE-90187 Umeå; ${ }^{3}$ Department of Oncology-Pathology, \\ Karolinska Institute, SE-171 76 Stockholm; ${ }^{4}$ Department of Laboratory Medicine, Division of Pathology, \\ Karolinska Institute, Karolinska University Hospital Huddinge, SE-141 86 Stockholm, Sweden
}

Received July 27, 2012; Accepted September 30, 2012

DOI: $10.3892 /$ ijo.2012.1702

\begin{abstract}
The incidence of cervical adenocarcinoma, which accounts for $10-20 \%$ of all cervical cancers, has increased continuously in developed countries during the last two decades, unlike squamous cell cervical carcinoma. This increasing trend, noted particularly among women under the age of 40 years, has occurred despite extensive cytological Pap smear screening. A deeper understanding of the etiology of cervical adenocarcinoma, better preventive measures and reliable prognostic markers are urgently needed. The human leucinerich repeats and immunoglobulin-like domains (LRIG) gene family includes: LRIG1, LRIG2 and LRIG3. LRIG expression has proven to be of prognostic value in different types of human cancers, including breast cancer, early stage invasive squamous cervical cancer, cutaneous squamous cell carcinoma, oligodendroglioma and astrocytoma. LRIG1 functions as a tumor suppressor, while less is known about the functions of LRIG2 and LRIG3. This study evaluated the expression of the three LRIG proteins in tumor specimens from 86 women with pure cervical adenocarcinoma by immunohistochemistry. Possible correlations between LRIG expression and known prognostic factors, including human papillomavirus (HPV) status, FIGO stage and histology were investigated.
\end{abstract}

Correspondence to: Dr Sonia Andersson, Department of Women's and Children's Health, Division of Obstetrics and Gynecology, Karolinska Institute, Elevhemmet H2:00, Karolinska University Hospital Solna, SE-171 76 Stockholm, Sweden

E-mail: sonia.andersson@karolinska.se

${ }^{*}$ Contributed equally

Key words: cervical adenocarcinoma, human papillomavirus, human leucine-rich repeats and immunoglobulin-like domains, survival
Patient survival data were collected retrospectively and the possible prognostic value of LRIG protein expression was investigated. High staining intensity of LRIG1 and high fraction of LRIG3-positive cells were significantly associated with patient survival, and positive correlations were found between LRIG1 and LRIG3 staining intensity and HPV status. Thus, the LRIG proteins may be important determinants of cervical adenocarcinoma progression and their diagnostic and prognostic potential should be studied further.

\section{Introduction}

Cervical cancer is the second most common cancer in women worldwide and approximately 529,000 cases are diagnosed each year (1). In Sweden, about 450 women are diagnosed with invasive cervical cancer annually and 150 die from the disease each year (2). In the last decade, an increase in incidence of invasive adenocarcinoma, which currently comprises 10-20\% of all cervical cancers, has been observed in several countries where cervical screening programs exist (3-5). In Stockholm county, Sweden, before screening was introduced the percentage of adenocarcinomas of all uterine cervical cancers varied between 2 and 7\% (6). During the screening period, the incidence of adenocarcinoma was higher and accounted for $25.7 \%$ of cases. In the screening cohort, a marked difference was observed between younger and older women; the percentage of adenocarcinomas among younger women was about 6 times higher and in women $\geq 70$ years about 3 times higher than in the prescreening cohort (6). In general, clinical screening programs that have led to a substantial decrease in the incidence of squamous cell carcinoma (SCC) have only had a limited protective effect on the occurrence of adenocarcinoma and its precursor lesion, adenocarcinoma in situ (7). The prognosis for cervical adenocarcinoma has also been reported to be slightly worse than for cervical SCC (8). Known prognostic factors include FIGO stage, tumor lesion size, and lymph node metastasis status (8). 
High-risk human papillomavirus (HPV) is an important etiological agent in the pathogenesis of cervical adenocarcinoma (9). HPV DNA is usually detected in 30-90\% of cervical adenocarcinomas, with HPV 18 as the predominant type $(10,11)$. Although closely linked to cervical cancer development, HPV infection alone is not sufficient for oncogenic transformation; additional factors are required for malignant transformation of normal cervical epithelium into adenocarcinoma (12).

The human leucine-rich repeats and immunoglobulinlike domains (LRIG) gene family includes: LRIG1, LRIG2 and LRIG3. LRIG1 has been shown to negatively regulate receptor tyrosine kinases, including members of the epidermal growth factor receptor family (13-15), MET (16) and RET (17). LRIG1 has been suggested to function as a tumor suppressor in various tissues (reviewed in ref. 18), and was recently shown to be a bona fide tumor suppressor in mouse intestine (19). Less is known about the functions of LRIG2 and LRIG3. High expression of LRIG1 correlates with better prognosis in breast cancer (20), early stage invasive squamous cervical cancer (21) and in cutaneous squamous cell carcinoma (22). In contrast, LRIG2 expression correlates with poor survival in oligodendroglioma (23) and in early stage squamous cervical cancer (24). Both LRIG1 and LRIG2 have been found to correlate with increasing grade of cervical intraepithelial neoplasia (25). Thus, LRIG proteins seem to play an important role in both precancerous lesions and invasive squamous cervical cancer, but the possible prognostic value of the LRIG proteins in cervical adenocarcinoma is largely unknown since it has only been studied in a small series of cases (21).

The aim of this study was to evaluate the immunohistochemical expression of the LRIG proteins in 86 cervical adenocarcinomas, and to investigate possible correlations between LRIG expression, patient survival and HPV status.

\section{Materials and methods}

Patients and tumor characteristics. This study looked at a cohort of 86 patients with primary cervical adenocarcinoma treated at Karolinska University Hospital, Huddinge, between 1992 and 2000. The study was approved by The Regional Ethics Review Board in Stockholm and all women provided written informed consent to participate in the study. All tumor cases were identified from the Swedish National Cancer Registry and were presented in a previous study (26). The previous study included 101 patients, while the current study conducted immunohistochemical analyses for the expression of LRIG proteins in 86 patients, after exclusion of 15 patients due to an insufficient amount of material.

The histopathological diagnoses were based on WHO criteria and the FIGO system was used for clinical classification. All tumors were cervical adenocarcinomas and lacked any squamous cell component; 79 were invasive, 4 were in situ and histopathological diagnosis could not be ascertained for the remaining 3 patients. Table I presents grade of differentiation. FIGO classification was obtained for 72 patients and Table I presents the distribution between stages. All patients were retrospectively followed up from time of diagnosis until January 2011 and survival data were recorded.
Table I. Patient and tumor characteristics.

Patient and tumor characteristics

\begin{tabular}{lc}
\hline Patients included in the study & 86 \\
Age at diagnosis (years) & 48 \\
Median & 51 \\
Mean & \\
FIGO stage (n=72) & 4 \\
0 & 52 \\
I & 10 \\
II & 6 \\
III & \\
Histopathological grade (n=79) & 35 \\
Well differentiated & 34 \\
Moderately differentiated & 10 \\
Poorly differentiated & \\
HPV status (n=86) & 57 \\
Positive & 29 \\
Negative & \\
Outcome (n=83) & 27 \\
Dead & 56 \\
Alive & \\
\hline
\end{tabular}

HPV status. Detailed descriptions of detection of HPV status and HPV screening analysis results were previously published (26). Briefly, DNA was extracted from a $10-\mu \mathrm{m}$ thick section of paraffin block and polymerase chain reaction (PCR) was used to amplify the L1 region of the HPV genome. Samples that yielded a PCR product of 130-150 base pairs that were visible after agarose gel electrophoresis and ethidium bromide staining were considered positive. Among the 86 cases included in this study, 57 (66\%) were HPV-positive and 29 (34\%) were HPV-negative. Of the HPV-positive tumors, 27 were infected with HPV 16, 25 with HPV 18 and 5 with HPV 45.

Immunohistochemistry for the LRIG proteins. Immunohistochemical staining of LRIG1, LRIG2 and LRIG3 was carried out using polyclonal rabbit antibodies against the cytosolic tails of the proteins, as previously described (27). Tissue sections containing squamous cervical cancer were used as positive controls, and appropriate negative controls were always included.

A senior pathologist (L.K.), blinded to all clinical data, evaluated the immune staining. Immunohistochemistry results were scored based on both staining intensity and percentage of immunoreactive epithelial cells. The percentage of positive cells was based on a 5-grade semi-quantitative scale: 0 , no expression; $1,>0$ and $<24 \% ; 2,25-49 \%$ positive cells; $3,50-74 \%$ positive cells; $4,>75 \%$ positive cells. Intensity of staining was evaluated on a four-grade semi-quantitative scale: 0 , no staining; 1 , weak; 2 , moderate; and 3 , intense $(28,29)$.

Statistical analysis. Associations between ordinal variables were tested using a $\chi^{2}$ test or Fisher's exact test. Clinical 
Table II. Staining intensity (A) and fraction of positive cells (B) of the LRIG immunohistochemical analysis.

\begin{tabular}{lccrr}
\hline & \multicolumn{4}{c}{ LRIG staining intensity, $\mathrm{n}(\%)$} \\
\cline { 2 - 5 } $\mathrm{A}$ & No staining & Weak & Moderate & Intense \\
\hline LRIG1 & $11(12.9)$ & $18(21.2)$ & $26(30.6)$ & $30(35.3)$ \\
LRIG2 & $5(5.9)$ & $19(22.4)$ & $34(40.0)$ & $28(31.8)$ \\
LRIG3 & $17(20.0)$ & $59(69.4)$ & $8(9.4)$ & $(1.2)$ \\
\hline
\end{tabular}

\begin{tabular}{|c|c|c|c|c|c|}
\hline \multirow[b]{2}{*}{ B } & \multicolumn{5}{|c|}{ Fraction of LRIG-positive cells, n (\%) } \\
\hline & 0 & $>0$ and $<24 \%$ & $25-49 \%$ & $50-75 \%$ & $>75 \%$ \\
\hline LRIG1 & $11(12.8)$ & $4(4.7)$ & $16(18.6)$ & $25(29.1)$ & $30(34.9)$ \\
\hline LRIG2 & $5 \quad(5.8)$ & $3(3.5)$ & $14(16.3)$ & $8(9.3)$ & $56(65.1)$ \\
\hline LRIG3 & $17(20.0)$ & $11(12.9)$ & $11(12.9)$ & $11(12.9)$ & $35(41.1)$ \\
\hline
\end{tabular}

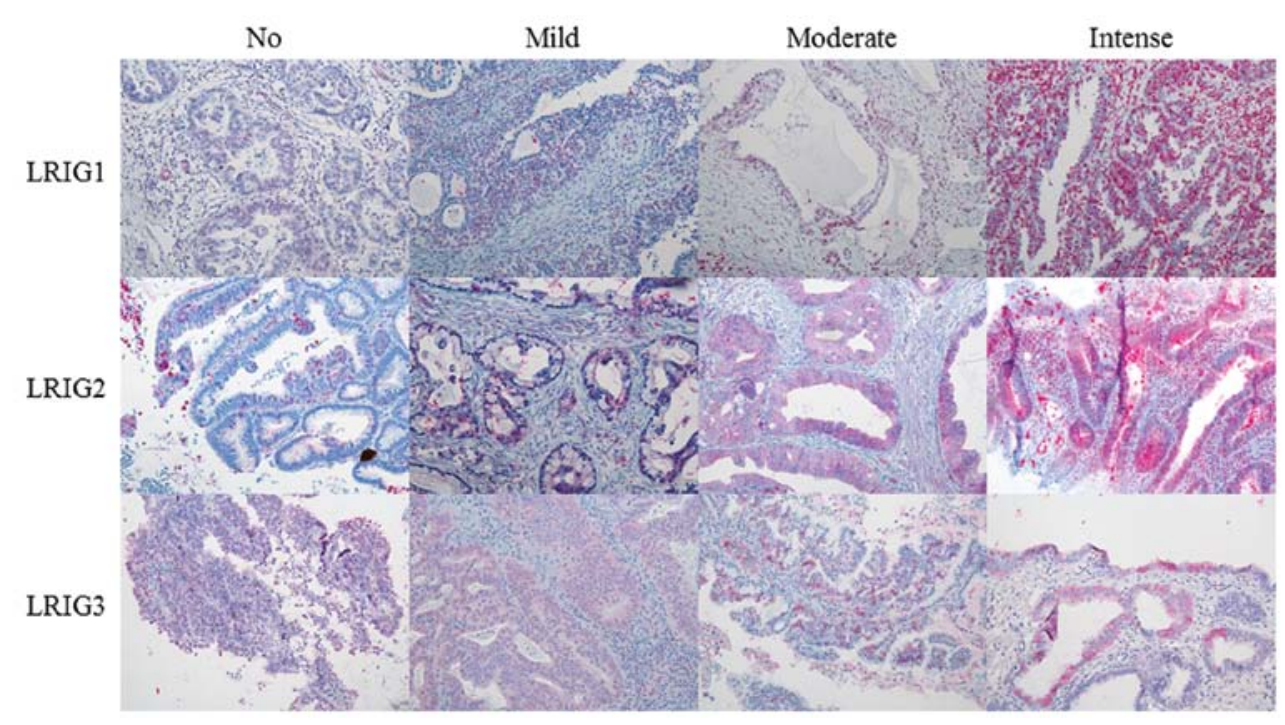

Figure 1. Examples of different staining intensities for LRIG1, LRIG2 and LRIG3 in cervical adenocarcinoma.

parameters, HPV status and expression of LRIG proteins were illustrated in a Kaplan-Meier graph to reflect possible prognostic factors for overall survival, and a log-rank test was used to compare different groups. The molecular markers found to be significant in the univariate analyses were considered in a Cox regression multivariate analysis, including age (young vs. old, dichotomized based on median age), histology and FIGO stage. All significance testing was carried out at the 0.05 level, using SPSS 19 software.

\section{Results}

Immunohistochemical expression of LRIG proteins and their correlation to HPV and clinical parameters. Evaluation of LRIG staining intensity and fraction of LRIG-positive cells was carried out for all 86 patients, except in one case each for LRIG1 and LRIG3 staining intensity, and fraction of LRIG3positive cells, where technical reasons precluded evaluation.
Table II presents evaluation of immunohistochemical staining, while Fig. 1 shows examples of the evaluation of staining intensity. Regarding staining intensity, LRIG1 and LRIG2 were distributed almost equally, in contrast to LRIG3, where most tumors showed either no staining or weak staining $(n=76)$. When considering the fraction of positive cells, the majority of cases demonstrated $>50 \%$ expressing cells for all three LRIG proteins. In the majority of cases, immunoreactivity of LRIG1 protein was seen only in the cytoplasm, LRIG2 immunoreactivity was expressed in both cytoplasm and the nucleus, while in LRIG3 protein, immunoreactivity was seen mostly in the nucleus.

HPV status showed significant correlation with LRIG1 and LRIG3 staining intensity $(\mathrm{P}=0.001$ and 0.004 , respectively; Table III), where high LRIG staining intensity correlated with HPV positivity. No other statistically significant correlations were found between LRIG staining intensity or fraction of positive cells, and HPV status, FIGO stage or histology, respectively. 
Table III. Correlation between HPV status and intensity of staining for LRIG1 (A) and LRIG3 (B), respectively ( $\mathrm{P}=0.03$ and 0.04 , respectively).

\begin{tabular}{|c|c|c|c|c|}
\hline \multirow[b]{2}{*}{ A } & \multicolumn{4}{|c|}{ LRIG1 staining intensity, n (\%) } \\
\hline & 0 & 1 & 2 & 3 \\
\hline $\mathrm{HPV}^{+}$ & $5 \quad(8.9)$ & $6(10.7)$ & $19(33.9)$ & $26(46.4)$ \\
\hline \multirow[t]{2}{*}{$\mathrm{HPV}^{-}$} & $6(20.7)$ & $12(41.4)$ & $7(24.1)$ & $4(13.8)$ \\
\hline & \multicolumn{4}{|c|}{ LRIG3 staining intensity, n (\%) } \\
\hline B & 0 & 1 & 2 & 3 \\
\hline $\mathrm{HPV}^{+}$ & $5 \quad(8.9)$ & $45(80.4)$ & $5(8.9)$ & $1(1.8)$ \\
\hline $\mathrm{HPV}^{-}$ & $12(41.4)$ & $14(48.3)$ & $3(10.3)$ & $0(0.0)$ \\
\hline
\end{tabular}

LRIG expression, HPV status, tumor stage and histology: correlation with patient survival. Reliable follow-up data were available for 83 patients. Median follow-up time was 12 years (range, 0-22 years) for all women, and 14 years (range, 10-22 years) for those women who did not die of cervical cancer. The association between staining intensity and fraction of cells positive for LRIG proteins, and survival, was estimated using a Kaplan-Meier plot and the groups were dichotomized using the median as a cut-off to generate two groups of equal size. Correlations between survival and HPV status, stage and histology, respectively, were also evaluated using the KaplanMeier method.

Both high staining intensity of LRIG1-positive cells and high fraction of LRIG3-positive cells correlated with survival ( $\mathrm{P}=0.03$ and 0.04, respectively; Fig. 2). A significant correlation was also found between HPV status and survival $(\mathrm{P}<0.001$, graph not shown). Survival data from these patients were previously published (30), but the patient group currently under study is slightly different, excluding 15 patients as
Table IV. Multivariate analysis including LRIG1 staining intensity, fraction of LRIG3-positive cells, HPV status, age, histology and FIGO stage.

\begin{tabular}{lccc}
\hline & HR & $(95 \% \mathrm{CI})$ & P-value \\
\hline LRIG1 intensity & 0.964 & $(0.407-2.285)$ & 0.934 \\
$\begin{array}{l}\text { Fraction of LRIG3 } \\
\text { positive cells }\end{array}$ & 0.401 & $(0.175-0.919)$ & 0.031 \\
HPV status & & & \\
Age & 0.447 & $(0.181-1.104)$ & 0.081 \\
Histology & 5.606 & $(1.847-17.015)$ & 0.002 \\
FIGO stage & 1.804 & $(0.994-3.274)$ & 0.052 \\
& 1.448 & $(0.902-2.322)$ & 0.125 \\
\hline
\end{tabular}

described above. Moreover, the patient group in the present study was followed until January 2011, compared with January 2007 in the previous study (30). As expected, both stage and histology also correlated with survival $(\mathrm{P}=0.02$ and $<0.001$, respectively, graph not shown). All significant parameters were then included in a Cox regression multivariate analysis (details presented in Table IV). The only factors that showed independent statistical significance in this analysis were age $(\mathrm{P}=0.002)$ and fraction of LRIG3-positive cells $(\mathrm{P}=0.031)$.

\section{Discussion}

In this study, LRIG protein immunoreactivity was evaluated in 86 cervical adenocarcinomas. Both high staining intensity of LRIG1-positive cells and a high fraction of LRIG3-positive cells were significantly associated with patient survival, and positive correlations were found between staining intensity of both LRIG1 and LRIG3 and HPV status. High expression of LRIG1 has previously been reported to correlate with better prognosis in breast cancer (20), early stage invasive squamous cervical cancer (21) and cutaneous squamous cell carcinoma (22). LRIG1 expression also correlates with increasing grade
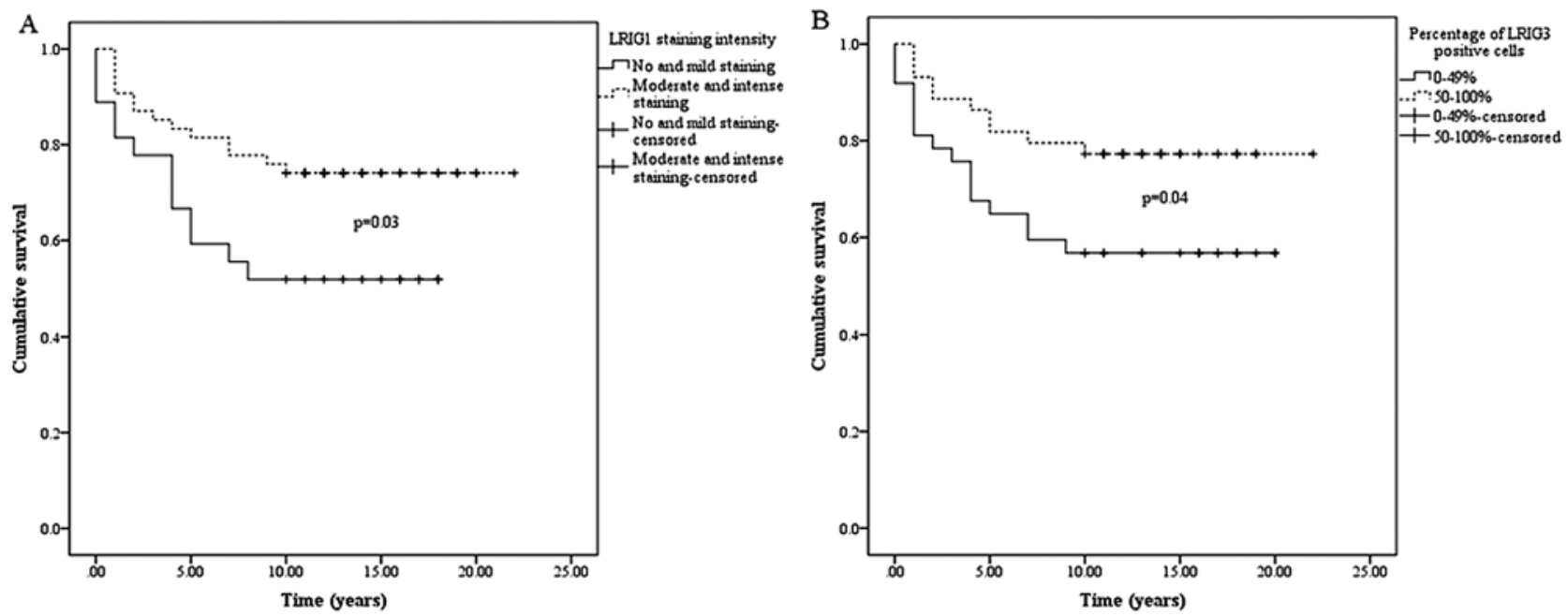

Figure 2. Correlation between survival and LRIG1 staining intensity (A) and fraction of LRIG3-positive cells (B), respectively. The 4 or 5 groups were dichotomized using the median as a cut-off to generate 2 groups of equal size. 
of cervical intraepithelial neoplasia. Thus, expression of LRIG1 seems to play an important role in both precancerous lesions and invasive squamous cervical cancer, and here we showed that LRIG1 also functions as a prognostic marker in cervical adenocarcinoma. One previous study investigated the immunoreactivity of LRIG1 in cervical adenocarcinoma. In this study, no correlation with survival was found, possibly due to the limited number of cases analyzed $(n=36)(21)$. The prognostic value of LRIG1 expression reported for cervical adenocarcinoma suggests its importance in this disease, as well as in other types of cancer. By negatively regulating receptor tyrosine kinases, it is possible that LRIG1 functions as a tumor suppressor in the uterine cervix.

Less is known about the functions of LRIG2 and LRIG3. LRIG2 expression has been reported to correlate with worse survival in cervical SCC (24); however, in the present study no correlation between LRIG2 expression and clinical parameters in cervical adenocarcinoma was found. Thus, LRIG2 might be more important as a possible marker of prognosis in cervical SCC and other cancer types. Immunohistochemical expression of LRIG3 has been evaluated in oligodendroglioma and in astrocytic tumors. Perinuclear staining of LRIG3 in astrocytic tumors correlated with better survival, which is consistent with the findings of the present study (27), suggesting that LRIG3 might have a tumor suppressive function similar to that of LRIG1.

The positive correlation between HPV status and LRIG1 and LRIG3 staining intensity is intriguing. The number of HPV positive cases $(66 \%)$ and distribution of HPV types are in line with that previously reported (31-33). We previously showed that HPV infection correlated with better outcome in the present study cohort (30), and this finding still holds after an additional four years of follow-up. The presence of two groups of cervical adenocarcinomas, one with positive and one with negative HPV status, is similar to the situation in tonsillar and base of tongue cancer, where there is increasing evidence for two different tumor types, depending on HPV status $(34,35)$. Whether this also holds true for cervical adenocarcinoma remains to be investigated, but our results suggest differences between HPV-positive and -negative cervical adenocarcinomas, at least in regard to LRIG expression and patient survival.

Since low expression of LRIG1 correlates with worse survival in cervical adenocarcinoma (Fig. 2A) and other human cancers (20-22), LRIG1 might be of interest as a potential target for treatment. It has been reported that LRIG1 ectodomains can be shed and suppress growth factor signaling in neighboring cells, suggesting that LRIG1 ectodomains can suppress cell proliferation in a paracrine manner. This non-cell autonomous inhibition of growth factor receptor signaling could be an interesting strategy for treatment of cervical adenocarcinoma and other cancers. However, whether cervical cancers depend on growth factor signaling for their growth and whether LRIG1 can inhibit this growth remain to be determined.

Cervical cancer screening programs have decreased mortality from cervical SCC in many developed countries. However, due to significant subjectivity and low sensitivity when interpreting Pap smears and cervical biopsy specimens, an active search for better biomarkers that may prove useful in clinical practice for early diagnosis of cervical adenocarcinomas and neoplastic glandular lesions is underway. Staging of adenocarcinoma based on morphology and histology is also difficult due to the lack of a clearly defined preinvasive phase. Thus, sensitivity for detecting precursor lesions of adenocarcinoma is much lower than that for SCC (36). A better understanding of the role of different molecular markers in the genesis of cervical adenocarcinoma is therefore important to optimize screening intervention. Molecular markers, such as the LRIG proteins studied here, may help us to develop more efficient screening strategies for adenocarcinoma of the cervix. Thus, analysis of LRIG expression in precursor lesions (i.e., adenocarcinoma in situ and glandular dysplasia) will be important to determine the potential of LRIG proteins as molecular markers for early detection and progression to invasive disease.

In summary, LRIG immunoreactivity is of prognostic importance in cervical adenocarcinoma and correlate with HPV status. Therefore, LRIG proteins may be important determinants of cervical adenocarcinoma progression, which justifies further study of their diagnostic and prognostic potential in this disease.

\section{Acknowledgements}

This study was supported by the Swedish Cancer Foundation (070623, CAN 2007/1044, 11 0544 CAN 2011/471), KI Cancer Strategic Grants (5888/05-722), Lion's Cancer Research Foundation, University of Umeå, Swedish Research Council (521-2008-2899), Medical Research Council and Cancer Society in Stockholm, Stockholm County Council.

\section{References}

1. Ferlay J, Shin HR, Bray F, Forman D, Mathers C and Parkin DM: Estimates of worldwide burden of cancer in 2008: GLOBOCAN 2008. Int J Cancer 127: 2893-2917, 2010.

2. Socialstyrelsen Ec: Cancer incidence in Sweden 2008, 2008.

3. Vizcaino AP, Moreno V, Bosch FX, Munoz N, Barros-Dios XM and Parkin DM: International trends in the incidence of cervical cancer: I. Adenocarcinoma and adenosquamous cell carcinomas. Int J Cancer 75: 536-545, 1998

4. Smith HO, Tiffany MF, Qualls CR and Key CR: The rising incidence of adenocarcinoma relative to squamous cell carcinoma of the uterine cervix in the United States - a 24-year populationbased study. Gynecol Oncol 78: 97-105, 2000.

5. Bray F, Carstensen B, Moller H, et al: Incidence trends of adenocarcinoma of the cervix in 13 European countries. Cancer Epidemiol Biomarkers Prev 14: 2191-2199, 2005.

6. Pettersson BF, Andersson S, Hellman K and Hellstrom AC: Invasive carcinoma of the uterine cervix associated with pregnancy: 90 years of experience. Cancer 116: 2343-2349, 2010.

7. Etherington IJ and Luesley DM: Adenocarcinoma in situ of the cervix-controversies in diagnosis and treatment. J Low Genit Tract Dis 5: 94-98, 2001.

8. Gien LT, Beauchemin MC and Thomas G: Adenocarcinoma: a unique cervical cancer. Gynecol Oncol 116: 140-146, 2010.

9. Bosch FX and Munoz N: The viral etiology of cervical cancer. Virus Res 89: 183-190, 2002.

10. Andersson S, Larson B, Hjerpe A, et al: Adenocarcinoma of the uterine cervix: the presence of human papillomavirus and the method of detection. Acta Obstet Gynecol Scand 82: 960-965, 2003.

11. Castellsague X, Diaz M, de Sanjose S, et al: Worldwide human papillomavirus etiology of cervical adenocarcinoma and its cofactors: implications for screening and prevention. J Natl Cancer Inst 98: 303-315, 2006.

12. Andersson S, Wallin KL, Hellstrom AC, et al: Frequent gain of the human telomerase gene TERC at $3 \mathrm{q} 26$ in cervical adenocarcinomas. Br J Cancer 95: 331-338, 2006.

13. Gur G, Rubin C, Katz M, et al: LRIG1 restricts growth factor signaling by enhancing receptor ubiquitylation and degradation. EMBO J 23: 3270-3281, 2004. 
14. Laederich MB, Funes-Duran M, Yen L, et al: The leucine-rich repeat protein LRIG1 is a negative regulator of ErbB family receptor tyrosine kinases. J Biol Chem 279: 47050-47056, 2004.

15. Miller JK, Shattuck DL, Ingalla EQ, et al: Suppression of the negative regulator LRIG1 contributes to ErbB2 overexpression in breast cancer. Cancer Res 68: 8286-8294, 2008.

16. Shattuck DL, Miller JK, Laederich M, et al: LRIG1 is a novel negative regulator of the Met receptor and opposes Met and Her2 synergy. Mol Cell Biol 27: 1934-1946, 2007.

17. Ledda F, Bieraugel O, Fard SS, Vilar M and Paratcha G: Lrig1 is an endogenous inhibitor of Ret receptor tyrosine kinase activation, downstream signaling, and biological responses to GDNF. J Neurosci 28: 39-49, 2008.

18. Hedman H and Henriksson R: LRIG inhibitors of growth factor signalling - double-edged swords in human cancer? Eur J Cancer 43: 676-682, 2007

19. Powell AE, Wang Y, Li Y, et al: The pan-ErbB negative regulator Lrig1 is an intestinal stem cell marker that functions as a tumor suppressor. Cell 149: 146-158, 2012.

20. Krig SR, Frietze S, Simion C, et al: Lrig1 is an estrogen-regulated growth suppressor and correlates with longer relapse-free survival in ERalpha-positive breast cancer. Mol Cancer Res 9: 1406-1417, 2011

21. Lindstrom AK, Ekman K, Stendahl U, et al: LRIG1 and squamous epithelial uterine cervical cancer: correlation to prognosis, other tumor markers, sex steroid hormones, and smoking. Int J Gynecol Cancer 18: 312-317, 2008.

22. Tanemura A, Nagasawa T, Inui S and Itami S: LRIG-1 provides a novel prognostic predictor in squamous cell carcinoma of the skin: immunohistochemical analysis for 38 cases. Dermatol Surg 31: 423-430, 2005.

23. Holmlund C, Haapasalo H, Yi W, et al: Cytoplasmic LRIG2 expression is associated with poor oligodendroglioma patient survival. Neuropathology 29: 242-247, 2009.

24. Hedman H, Lindstrom AK, Tot T, Stendahl U, Henriksson R and Hellberg D: LRIG2 in contrast to LRIG1 predicts poor survival in early-stage squamous cell carcinoma of the uterine cervix. Acta Oncol 49: 812-815, 2010.

25. Lindstrom AK, Asplund A and Hellberg D: Correlation between LRIG1 and LRIG2 expressions and expression of 11 tumor markers, with special reference to tumor suppressors, in CIN and normal cervical epithelium. Gynecol Oncol 122: 372-376, 2011.
26. Andersson S, Rylander E, Larson B, et al: Types of human papillomavirus revealed in cervical adenocarcinomas after DNA sequencing. Oncol Rep 10: 175-179, 2003.

27. Guo D, Nilsson J, Haapasalo H, et al: Perinuclear leucine-rich repeats and immunoglobulin-like domain proteins (LRIG1-3) as prognostic indicators in astrocytic tumors. Acta Neuropathol 111: 238-246, 2006.

28. Klussmann JP, Gultekin E, Weissenborn SJ, et al: Expression of p16 protein identifies a distinct entity of tonsillar carcinomas associated with human papillomavirus. Am J Pathol 162: 747-753, 2003.

29. Sano T, Oyama T, Kashiwabara K, Fukuda T and Nakajima T. Expression status of p16 protein is associated with human papillomavirus oncogenic potential in cervical and genital lesions. Am J Pathol 153: 1741-1748, 1998

30. Muller S, Flores-Staino C, Skyldberg B, et al: Expression of p16INK4a and MIB-1 in relation to histopathology and HPV types in cervical adenocarcinoma. Int J Oncol 32: 333-340, 2008.

31. Seoud M, Tjalma WA and Ronsse V: Cervical adenocarcinoma: moving towards better prevention. Vaccine 29: 9148-9158, 2011.

32. Tenti P, Romagnoli S, Silini E, et al: Human papillomavirus types 16 and 18 infection in infiltrating adenocarcinoma of the cervix: PCR analysis of 138 cases and correlation with histologic type and grade. Am J Clin Pathol 106: 52-56, 1996.

33. Andersson S, Rylander E, Larsson B, Strand A, Silfversvard C and Wilander E: The role of human papillomavirus in cervical adenocarcinoma carcinogenesis. Eur J Cancer 37: 246-250, 2001.

34. Attner P, Du J, Nasman A, et al: Human papillomavirus and survival in patients with base of tongue cancer. Int J Cancer 128: 2892-2897, 2011

35. Hammarstedt L, Lindquist D, Dahlstrand H, et al: Human papillomavirus as a risk factor for the increase in incidence of tonsillar cancer. Int J Cancer 119: 2620-2623, 2006.

36. Krane JF, Granter SR, Trask CE, Hogan CL and Lee KR Papanicolaou smear sensitivity for the detection of adenocarcinoma of the cervix: a study of 49 cases. Cancer 93: 8-15, 2001. 